\title{
Diverticular disease in young adults
}

\author{
THOMAS W. WEHMANN, DO
}

VAL A. RONGAUS, DO

Acute diverticulitis of the colon should be considered in the differential diagnosis in young adult (younger than 40 years) patients with pain and tenderness in the lower abdomen. Among young adults, men are afflicted more commonly than women and the disease runs a more virulent course. Complications are commonly encountered, and the key to diagnosis is a high index of suspicion. Barium enema examination without bowel preparation is useful and safe in this age group. In patients with acute diverticulitis with perforation and abscess, the most appropriate therapy is surgical, as demonstrated in the three cases reported here.

Diverticular disease of the colon usually is considered a disease of older adults, rarely seen in patients younger than the age of 40 . In cases in which a younger person is found to have the disease, the diagnosis usually is not made immediately, and the disease typically runs a more virulent course. ${ }^{1}$ In younger patients, diverticular disease usually is seen in males. The early onset of the disease and its characteristically more severe inflammatory course may be caused by an unusual defect in the colon wall or a more severe anatomic problem in the sigmoid colon. ${ }^{2}$

The most important step in making the correct diagnosis is simply to consider the possibility of diverticulitis when a young patient is suffering from pain and tenderness in the lower abdomen. ${ }^{3}$ To ensure early diagnosis of and appropriate therapy for diverticular disease, it is essential to make a complete differential diagnosis in cases in which the patient complains of abdominal pain.
In this article, we present three cases of acute diverticulitis seen in young adults in the course of 1 year at an osteopathic community hospital.

\section{Report of cases \\ Case 1}

A 36-year-old man was admitted with a 1-day history of nausea, low abdominal pressure, and pain in the lower right quadrant of the abdomen. This patient had a history of malignant brain tumor, mononucleosis, and hemorrhoids. He took Lomotil (diphenoxylate hydrochloride with atropine sulfate), one tablet three times a day. The family history was noncontributory. Vital signs were as follows: blood pressure, 158/80 $\mathrm{mm} \mathrm{Hg}$; heart rate, 80 beats per minute; respiratory rate, $22 / \mathrm{min}$; and temperature, $37^{\circ} \mathrm{C}$. The abdomen was soft and tender diffusely but more so in the lower right quadrant. There were no bowel sounds. There was a positive McBurney's sign and no rebound. The rectal examination revealed no stools, masses, or tenderness.

The WBC count was $14,600 / \mathrm{cu} \mathrm{mm}$, with 80 segmented neutrophils and 0 band neutrophils.

The admitting diagnosis was acute appendicitis.

At laparotomy, acute diverticulitis of the sigmoid colon, with walled-off pericolic access, was found. The patient underwent a Mikulicz's exteriorization procedure, with double-barrel colostomy. The postoperative course was uncomplicated, and the patient was discharged on the 8 th postoperative day. Closure of the colostomy was performed without complications at 8 weeks.

\section{Case 2}

A 36-year-old man was admitted with 1-day history of anorexia, nausea, chills, and suprapubic and periumbilical pain. The patient had no significant medical or surgical history, and the family history was noncontributory. Vital signs were as follows: blood pressure, 108/64 $\mathrm{mm} \mathrm{Hg}$; heart rate, 128 beats per minute; respiratory rate, $32 / \mathrm{min}$; and temperature $38^{\circ} \mathrm{C}$.

The abdomen was tender in the lower right quadrant and suprapubic regions. Rebound, guarding, and a positive Rovsing's sign were present. The stool examination 
was heme negative. The WBC count was $15,400 / \mathrm{cu} \mathrm{mm}$, with 72 segmented neutrophils, 12 band neutrophils, and 10 lymphocytes. The admitting diagnosis was acute appendicitis instead of possible acute diverticulitis with perforation.

At laparotomy, acute diverticulitis of the sigmoid colon, with perforation and abscess, was found. The patient underwent a Hartmann's procedure (resection of involved colon, end colostomy, and closure of the distal aspect of the rectum) along with drainage of the abscess. The postoperative course was uncomplicated, and the patient was discharged on the 6 th postoperative day. At 7 weeks, the patient had restoration of bowel continuity without complications.

\section{Case 3}

A 30-year-old obese man was admitted with a 3-day history of abdominal pain, nausea, vomiting, chills, and fever. At another institution, the patient had been placed on tetracycline therapy (500 $\mathrm{mg} \mathrm{q6h}$ ) for suspected gastroenteritis. The medical and surgical histories as well as the family history were noncontributory.

Vital signs were as follows: blood pressure, $110 / 58 \mathrm{~mm}$ $\mathrm{Hg}$; heart rate, 124 beats per minute; respiratory rate, $32 / \mathrm{min}$; and temperature, $39^{\circ} \mathrm{C}$. The abdomen was distended, soft, without bowel sounds, and with generalized tenderness, rebound, guarding, and distension. The stool examination was heme negative. The WBC count was $8,500 / \mathrm{cu} \mathrm{mm}$, with 44 segmented neutrophils, and 40 band neutrophils. The admitting diagnosis was acute appendicitis instead of diverticulitis.

At laparotomy, the patient was found to have acute diverticulitis of the sigmoid colon, with perforation and free intraperitoneal pus. The patient underwent a Hartmann's resection procedure and wide peritoneal drainage. The postoperative course was uncomplicated, and the patient was discharged on the 14th postoperative day. In 6 weeks, the patient had restoration of bowel continuity without complications.

\section{Discussion}

Traditionally, diverticular disease of the colon has been considered a disease of the older adult. In a review of 2,000 barium enema studies, Welch and his associates ${ }^{4}$ found no cases of diverticulitis in patients younger than 35 years. Of 4,673 patients admitted to the University of Rochester (NY) Medical Center with diverticulitis of the colon, only 115 $(2.5 \%)$ were younger than 40 years. ${ }^{5}$ In a study of 521 cases by Parks, ${ }^{6} 4.8 \%$ of the patients were younger than 40 years, and only $0.6 \%$ were younger than 39 years when they first complained of symptoms that suggested diverticular disease. Our three patients were treated in a community hospital within the period of 1 year.
Often the correct diagnosis of diverticulitis is not considered initially, and this may lead to a delay in treatment. Some researchers ${ }^{1,3}$ report that the correct diagnosis is initially made in only $25 \%$ to $35 \%$ of the cases.

Whether it is the delay in diagnosis that accounts for the apparent virulence of the disease in young adults is a source of controversy. Oriel and associates ${ }^{5}$ reported that an operative procedure was required in $30 \%$ of the total number of cases of diverticular disease but that it was required in $60 \%$ of the cases involving young patients. In their review of the literature of the past 50 years, Halata and associates ${ }^{7}$ found only 12 cases of diverticular disease in patients younger than 21 years. In 8 of these 12 cases, surgical intervention was required. Freischlag and associates ${ }^{1}$ reported that patients younger than 40 years required surgery significantly more frequently than those older than 40 years $(76.5 \%$ in contrast with $25 \%$ ).

In young patients, perforation and abscess formation appear to be the most frequent complications that lead to operative intervention. In such cases, most authorities ${ }^{1}$ would agree that aggressive management is warranted. In the three cases we describe, the patients had potentially life-threatening complications. In their study of diverticular disease in young patients, Eusabio and Eisenberg 8 found that in approximately $67 \%$ of the cases, surgery was necessary within 4 years of the initial attack. Simonowitz and Paloyan, ${ }^{9}$ however, found that only 4 of 24 young patients (about 17\%) who were treated medically and followed up for an average of 51.8 months required continued medical treatment and that none required surgical intervention.

In cases in which perforation and abscess are present, Hartmann's procedure usually is performed, with drainage of the abscess. Bowel continuity can be safely restored in 2 to 3 months. ${ }^{10,11}$ In the three cases we describe, the patients did well with their colostomy procedures and were restored to a normal life-style in a relatively short time.

The most important factor in making a correct diagnosis of acute diverticulitis in a young person complaining of pain and tenderness in the lower part of the abdomen is to suspect the disease..$^{1,3}$ Plain films of the abdomen have not been diagnostic; and fever and WBC count do not differentiate acute diverticulitis from other disorders. Frieschlag and colleagues showed that in cases in which young patients had uncommon or confusing peritoneal signs, barium enema examinations facili- 
tated the diagnostic workup and were useful in determining therapy. In 10 of 15 patients who had barium enema examinations, irritability, spasms, or perforations were found, and no untoward complications of the procedure were noted. ${ }^{1}$

In most studies of diverticular disease in older adults, the subjects are predominately women; in young adults, predominately men. ${ }^{5,12}$ The three patients described in this report were men.

Some investigators ${ }^{2}$ have suggested that patients in the younger age group may have an unusual defect in the colon wall or a more severe anatomic problem in the sigmoid colon that causes the early onset of the disease and its typically more severe inflammatory course. Diverticular disease also has been described in young patients with Marfan's syndrome, suggesting that the predisposing factor is an elastic collagen defect. ${ }^{13,14}$

It is important for the surgeon to consider acute diverticulitis in the differential diagnosis of abdominal pain in young adults. In young adults, appendicitis is much more common than diverticulitis. If the traditional incision at McBurney's point is made at laparotomy, the surgeon will have much difficulty in appropriately performing colostomy and drainage when acute diverticulitis with perforation is found. If the diagnosis of diverticulitis is considered, a barium enema examination without bowel preparation can be made, and an appropriate incision, probably midline, can be planned.

\section{Summary}

It is possible for young adults to have acute diverticulitis of the colon. Among young adults, men are more commonly afflicted than women, and the disease appears to be more virulent. It often is accompanied by perforation and abscess. Consideration of the possibility of diverticulitis is the most important factor in making the diagnosis. In difficult diagnostic cases, a barium or Gastrografin (meglumine diatrizoate) enema examination without bowel preparation is useful and safe. Recently, computed tomography scanning with Gastrografin has been used in difficult diagnostic cases. In cases of diverticulitis with perforation and abscess, the most appropriate therapy appears to be Hartmann's procedure, with removal of the involved segment, drainage of the abscess, and subsequent restoration of bowel continuity.

We have presented three cases seen in an osteopathic community hospital in the course of 1 year and pointed to the need for a full differential diagnosis in all cases of abdominal pain and of the in- clusion of acute diverticulitis in the differential diagnosis, regardless of the age of the patient.

1. Freischlag J, Bennion RS, Thompson JE: Complications of diverticular disease of the colon in young people. Dis Colon Rectum 1986;29:639. 643.

2. Johnson NC: Recurrent abdominal pain in a healthy 35-year-old. Hosp Prac 1982;17:97-100.

3. Chodak GW, Decio MR, Passaro E: Colonic diverticulitis in patients under age 40: Need for earlier diagnosis. Am J Surg 1981;141:699-701.

4. Welch CE, Allen AW, Donaldson LA: An appraisal of resection of the colon for diverticulitis of the sigmoid. Ann Surg 1983;138:332-343.

5. Oriel K, Schwartz SI: Diverticulitis in the young patient. Surg Gynecol Obstet 1983;156:1-5.

6. Parks TG: Natural history of diverticular disease of the colon: A review of 521 cases. $B r$ Med $J$ 1969;169:639-642.

7. Halata MS, Newman LJ, Easton LB, et al: Diverticulitis in an adolescent. Clin Pediatr (Phila) 1963;22:716-718.

8. Eusabio EB, Eisenberg MM: Natural history of diverticular disease of the colon in young patients. Am J Surg 1973;125:308-311.

9. Simonowitz D, Paloyan D: Diverticular disease of the colon in patients under 40 years of age. Am J Gastroenterol 1977;67:69-72.

10. Auguste L, Borrero E, Wise L: Surgical management of perforated colonic diverticulitis. Arch Surg 1985;120:450-452.

11. Killingback M: Management of perforative diverticulitis. Surg Clin of North Am 1983;63:97-115.

12. Rodkey GV, Welch CE: Changing patterns in the surgical management of diverticular disease. Ann Surg 1984;200:466-478.

13. Clunie GJA, Mason JM: Visceral diverticula and the Marfan syndrome. $B r$ J Surg 50:50-52.

14. Mielke JE, Becker KL, Gross JB: Diverticulitis of the colon in a young man with Marfan's syndrome. Gastroenterology 1965;48:379-382.

From the department of surgery, Cuyahoga General Hospital, Cuyahoga Falls, Ohio.

Reprint requests to Dr Wehmann, 2495 Northland St, Cuyahoga Falls, OH 44221. 